\title{
Using Life Cycle Assessment to identify potential environmental impacts of an agrifood sector: Application to the PDO Beaujolais and Burgundia wine sector
}

\author{
Sophie Penavayre ${ }^{1}$, Valérie Lempereur ${ }^{1}$, André-Paul Huet ${ }^{1}$, Sarah Gillet $^{2}$, Claire Pernet $^{2}$, Antoine Besnier ${ }^{3}$, \\ Laura Farrant ${ }^{4}$, et Franck Jolibert ${ }^{5}$ \\ ${ }^{1}$ Institut Français de la Vigne et du Vin (IFV), Pôle Bourgogne-Beaujolais-Savoie-Jura, 69661 Villefranche-sur-Saône, France \\ ${ }^{2}$ Bureau Interprofessionnel des Vins de Bourgogne (BIVB), 21200 Beaune, France \\ ${ }^{3}$ Institut des Corps Gras (ITERG), 33600 Pessac, France \\ ${ }^{4}$ Centre Technique de la Conservation des Produits Agricoles (CTCPA), 32000 Auch, France \\ ${ }^{5}$ Union Nationale des Groupements de Distillateurs d'Alcool (UNGDA), 92247 Malakoff, France
}

\begin{abstract}
The environmental impacts of the production system of emblematic French product under official quality marks was investigated using the Life Cycle Assessment (LCA) methodology. The study looks at the PDO Beaujolais and Burgundy sector from a broad perspective, i.e. encompassing all steps linked with the products themselves but also complementary activities that belong to this wine sector. To build the Life Cycle Inventory (LCI), a methodology deriving from both product and organizational LCA was developed and applied. The LCI was built using a bottom-up approach. Inventories were first built for a sample of 17 representative companies. Then, these inventories were scaled-up to complete the global LCI at the agrifood sector level. Potential environmental impacts were assessed for 8 indicators. The LCA results show potential environmental impacts for each life cycle step: grape production, wine making and aging, packaging, distribution and activity of stakeholders belonging to the "close environment". It provided two main outcomes: (i) a methodology for the construction of an LCI adapted to the perimeter of an agrifood sector and composed by high quality data; and (ii) the identification of potential environmental impacts of the studied agrifood sector, providing assistance for the definition of their strategic orientations for the future.
\end{abstract}

\section{Introduction}

L'Analyse de Cycle de Vie (ACV) est une méthode d'évaluation des impacts environnementaux potentiels d'un bien ou d'un service. L'ACV est multi-étapes (étudie les impacts de la fabrication d'un produit tout au long de son cycle de vie, de l'extraction des matières premières à la gestion des déchets) et multi-critères (exprime les dommages sur la santé humaine, les écosystèmes ou encore les ressources naturelles). Développée pour étudier un bien ou un service (cf. série des normes ISO 14040 à 14044 [1]), elle est l'objet de projets de recherche qui visent à l'adapter à l'évaluation des impacts environnementaux de périmètres différents comme celui des entreprises (ACV des organisations [2]) ou encore celui des territoires [3].

Parmi ces projets de recherche, ACYDU, co-financé par l'Agence Nationale de la Recherche (ANR), a pour objectif de définir une méthode permettant d'évaluer la durabilité des filières agroalimentaires. ACYDU étudie ainsi les impacts et performances environnementaux, sociaux, économiques et sur les territoires des filières de production de trois produits français emblématiques sous signe de qualité : Indication Géographique Protégée (IGP) Foie Gras du Sud-ouest, vins Appellation d'Origine Protégée (AOP) de Beaujolais et Bourgogne et AOP Comté. Les impacts environnementaux sont étudiés via la méthode de l'ACV, adaptée à la spécificité du projet: l'approche filière.

En effet, bien que les produits agroalimentaires, dont les vins, aient été l'objet de plusieurs études d'impacts environnementaux par ACV, aucune étude n'a étudié une filière agroalimentaire. L'ACV réalisée en 2013 par Neto, Dias et Machado [4] par exemple, étudie la chaîne de production d'un vin blanc produit sous AOP au Portugal, le vinho verde. Les étapes prises en compte sont la viticulture, l'étape de vinification-élevageconditionnement et la distribution des vins. Un focus est également fait sur la production des bouteilles de verre. L'inventaire a été réalisé auprès d'une seule entreprise produisant $25 \%$ des volumes de vinho verde. Un autre exemple est l'ACV de Petti et al. [5] qui étudie la «chaîne de production » (de la viticulture à la distribution du vin) d'un vin produit en agriculture biologique, sous appellation, en Italie. L'inventaire est réalisé auprès d'une exploitation de 12 ha.

L'objectif de ce travail d'évaluation environnementale à l'échelle de filières est double : proposer d'une part des adaptations méthodologiques à l'ACV permettant d'étudier non plus un produit ou une organisation mais l'ensemble d'une filière ; et identifier d'autre part les impacts environnementaux des trois filières étudiées offrant ainsi un support aux responsables des filières pour la définition de leurs stratégies d'amélioration. 
Cet article présente l'approche méthodologique développée pour l'Analyse Environnementale d'une Filière $(\mathrm{AEF})$ et les résultats obtenus pour la filière des Vins AOP de Beaujolais et Bourgogne.

Les vins AOP de Beaujolais et Bourgogne sont produits sur le territoire de la «Grande Bourgogne », sur une surface cultivée de plus de 46000 ha. La production vitivinicole est très majoritairement associée à des AOP qui peuvent être spécifiques au Beaujolais (12 AOP dont 10 crus), à la Bourgogne (plus de 100 AOP dont des appellations régionales, des appellations villages, des premiers crus et des grands crus), ou encore produites sur l'ensemble du territoire (5 AOP). Au cours de la campagne 2011-2012 (campagne viticole étudiée dans le cadre du projet ACYDU), plus de $2371000 \mathrm{hl}$ de vins (tranquilles et effervescents) ont été produits en AOP.

\section{Méthode}

\subsection{Détermination d'un périmètre spécifique : la filière}

La méthode de l'ACV, dès lors qu'elle étudie un produit, bien ou service, ou encore une organisation, dispose de normes internationales $[1,2]$ permettant de guider sa réalisation et d'assurer la cohérence des résultats. Les développements méthodologiques réalisés dans le cadre du projet ACYDU sont liés à la nécessité de travailler à l'échelle spécifique de la filière agroalimentaire. Aucune norme ne définit pour le moment comment réaliser une $\mathrm{ACV}$ ayant comme périmètre une filière.

Le périmètre de la filière agroalimentaire a préalablement été défini par un travail méthodologique également réalisé dans le cadre du projet ACYDU [6,7].

La filière est constituée :

- d'une « filière de production et de distribution » composée d'un cœur de filière dans lequel on trouve les entreprises et organisations directement impliquées dans la réalisation du produit principal auquel s'ajoutent les acteurs de l'amont (pépiniéristes, fournisseurs d'équipements et d'intrants viti et vini, verriers, bouchonniers, sous-traitants, etc.) et les acteurs de l'aval (distribution, consommateurs, distilleries, etc.),

- d'un « environnement proche » comprenant les structures « supports»: organismes de gestion et de défense, syndicats, interprofessions, organismes de recherche et développement, etc.

La réunion de l'ensemble des activités de ces structures compose le périmètre étudié dans le cadre du projet ACYDU, appelé « filière ». La Fig. 1 représente l'ensemble des acteurs de la filière. L'unité fonctionnelle, correspondant aux flux de référence auxquels sont rapportés les impacts au moment de l'expression des résultats, est déterminée en fonction du périmètre étudié. L'unité fonctionnelle retenue est l'activité de la filière des Vins AOP de Beaujolais et Bourgogne au cours d'un cycle de production, la campagne viticole 2011-2012.

\subsection{Construction d'un Inventaire de Cycle de Vie de qualité à l'échelle de la filière}

Le périmètre de la filière comprend plusieurs étapes du cycle de vie du vin (viticulture, vinification, élevage, conditionnement, distribution et consommation). $\mathrm{Au}$ sein de chaque étape, plusieurs catégories d'acteurs interviennent, composées chacune par un grand nombre d'entreprises.

La construction d'un Inventaire de Cycle de Vie (ICV) de la filière passe alors par une phase de collecte de données, dont la méthode varie en fonction de l'étape du cycle de vie et des catégories d'acteurs agissant au sein de chaque étape [8].

\subsubsection{ICV de la viticulture}

Pour la phase viticole, les données utilisées proviennent de la base de données d'ICV AGRIBALYSER [9]. Cette base de données, créée par l'Agence pour le DEveloppement et la Maîtrise de l'Energie (ADEME) met à disposition des ICV de produits agricoles français afin d'évaluer leurs impacts environnementaux potentiels. AGRIBLAYSERContient plusieurs ICV de production de raisins de cuve dont deux ancrés dans la filière étudiée : un ICV de production de raisins de cuve en AOP Beaujolais selon un système conventionnel et un ICV en AOP Mâconnais suivant un itinéraire de production en agriculture biologique.

\subsubsection{ICV de la vinification-élevage et du conditionnement}

Pour les étapes du cycle de vie liées à la transformation des raisins (vinification et élevage) et au conditionnement des vins, une collecte de données spécifique a été réalisée auprès d'entreprises identifiées comme représentatives de la filière.

A partir de l'identification des différentes catégories d'acteurs (cf. Fig. 1), un plan de collecte a été établi afin de définir les critères de représentativité des entreprises. Trois critères principaux ont été définis : le type d'entreprise (cave particulière, cave coopérative ou négociant), le volume vinifié (ou commercialisé pour les négociants) et la localisation (Beaujolais ou Bourgogne). Des ICV ont pu être constitués auprès de 17 entreprises répondant à ces critères. Les données collectées correspondent aux flux entrants et sortants des sites de production (énergies, eau, intrants œnologiques et de nettoyage, déchets et coproduits), distances et modes de transport pour l'acheminement sur site des raisins de cuve, des intrants et le transport des coproduits vers la distillerie. La distillation des coproduits (marcs de raisins et lies de vin) est intégrée à l'étape de vinification élevage. Les données de l'inventaire ont été collectées auprès de la distillerie du territoire.

Les données collectées auprès des 17 entreprises ont ensuite été agrégées et extrapolées pour constituer l'ICV à l'échelle de la filière. Le volume de vin produit a été choisi comme critère d'extrapolation. En effet, issu des déclarations de récolte complétées annuellement par les opérateurs, et utilisé par les interprofessions pour calculer la production totale de la filière, le volume est considéré comme une valeur très fiable. La répartition des volumes vinifiés et conditionnés, à l'échelle de la filière, par les trois types d'acteurs considérés (cave particulière, cave coopérative ou négociant) a été calculée à partir de données issues des interprofessions (cf. Tableau 1). Les entreprises ont été regroupées en 6 classes, en fonction de leur 


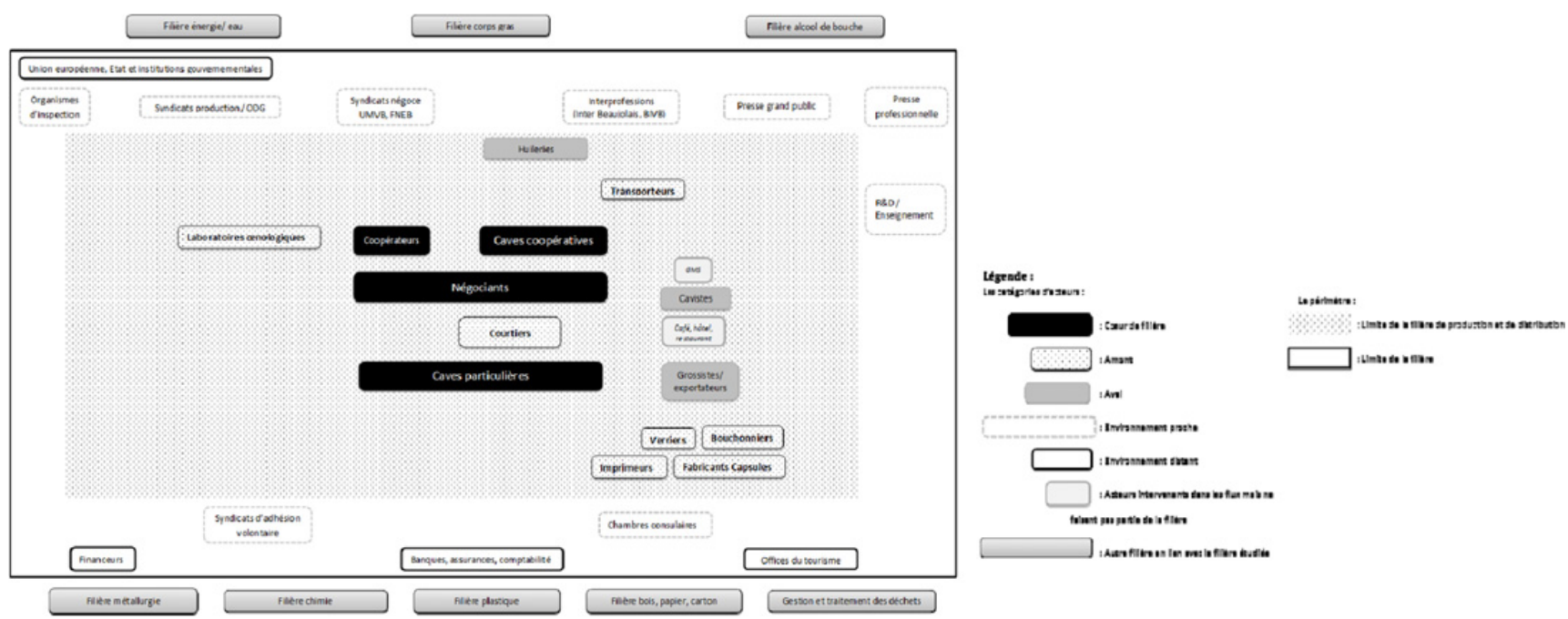

Figure 1. Schéma de la filière des vins AOP de Beaujolais et de Bourgogne.

Tableau 1. Répartition des volumes vinifiés-élevés et conditionnés par types d'entreprises pour la filière des vins AOP de Beaujolais et Bourgogne au cours de la campagne 2011-2012.

\begin{tabular}{|l|c|c|}
\hline $\begin{array}{l}\text { Types } \\
\text { d'entreprises }\end{array}$ & $\begin{array}{c}\text { Volume } \\
\text { vinifié-élevé (\%) }\end{array}$ & $\begin{array}{c}\text { Volume } \\
\text { conditionné(\%) }\end{array}$ \\
\hline Caves particulières & 54 & \multirow{2}{*}{35} \\
\hline Caves coopératives & 31 & \\
\hline Négociants & 15 & 65 \\
\hline
\end{tabular}

Tableau 2. Calcul du facteur de multiplication pour agréger et extrapoler les ICV des entreprises vers l'ICV de la filière, pour l'étape de vinification-élevage.

\begin{tabular}{|c|c|c|c|c|}
\hline \multicolumn{2}{|c|}{$\begin{array}{c}\text { Classes } \\
\text { d'entreprises } \\
\text { (types } \\
\text { d'entreprises et } \\
\text { localisation) }\end{array}$} & $\begin{array}{c}\text { Volume } \\
\text { total de la } \\
\text { classe à } \\
\text { l'échelle } \\
\text { de la filière } \\
\text { (hl) }\end{array}$ & \begin{tabular}{|c|} 
Volume \\
représenté \\
par les \\
17 \\
entreprises \\
ACYDU (h)
\end{tabular} & $\begin{array}{c}\text { Facteur } \\
\text { d'extrapolation }\end{array}$ \\
\hline \multirow{2}{*}{$\begin{array}{l}\text { Cave } \\
\text { particulière }\end{array}$} & Beaujolais & 534969 & 1768 & 303 \\
\hline & Bourgogne & 740304 & 4800 & 154 \\
\hline \multirow{2}{*}{$\begin{array}{l}\text { Cave } \\
\text { coopérative }\end{array}$} & Beaujolais & 292740 & 82128 & 3,5 \\
\hline & Bourgogne & 453000 & 112989 & 4 \\
\hline Négociant & $\begin{array}{l}\text { Beaujolais } \\
\text { Bourgogne }\end{array}$ & $\begin{array}{c}33291 \\
316696\end{array}$ & $\begin{array}{c}4897 \\
13039\end{array}$ & $\begin{array}{l}6,8 \\
24\end{array}$ \\
\hline
\end{tabular}

type et de leur localisation. Les ICV des entreprises ont été agrégés selon ces 6 classes. Pour l'extrapolation à l'échelle de la filière, un facteur d'extrapolation a été défini pour chaque classe d'entreprise. Les ICV agrégés ont été multipliés par ce facteur, puis sommés pour obtenir les ICV de la vinification-élevage et du conditionnement des vins, à l'échelle de la filière (cf. Tableau 2).

\subsubsection{ICV de la distribution des vins}

L'ICV de l'étape de distribution des vins a été principalement constitué à partir de données génériques collectées auprès des interprofessions du Beaujolais et de la Bourgogne : circuits de vente (grande distribution, café-hôtels-restaurants ou vente directe) et volumes de vins distribués par régions françaises et par pays pour la part exportée. Au transport des vins s'ajoutent l'ICV du stockage chez le distributeur et sur le lieu de vente (consommations énergétiques et d'eau), ainsi que les déplacements professionnels des salariés en charge de la commercialisation des vins.

\subsubsection{ICV de la consommation des vins}

L'ICV de l'étape de consommation est constitué à partir de données semi-spécifiques et génériques, notamment issues de la démarche européenne PEFCR en cours sur le vin [10]. Il comprend le transport des vins jusqu'au lieu de consommation, leur stockage et la fin de vie des emballages primaires.

\subsubsection{ICV de l'environnement proche}

Pour l'environnement proche, l'ICV est réalisé à partir de données spécifiques collectées auprès d'un échantillon d'entreprises appartenant à cette catégorie d'acteurs. L'agrégation et l'extrapolation à l'échelle de la filière ont été réalisées sur le critère du nombre d'Equivalent Temps Plein (ETP) de chaque entreprise enquêtée par rapport au nombre d'ETP de chaque catégorie d'acteurs à l'échelle de la filière.

\subsection{Analyse de Cycle de Vie}

Les impacts environnementaux potentiels ont été étudiés sur 8 indicateurs :

- le changement climatique (émissions de $\mathrm{CO}_{2}$ ),

- la diminution des ressources minérales et fossiles (quantité consommée par rapport à la disponibilité),

- la consommation d'eau (volume d'eau prélevé),

- les émissions de particules (conséquences sur la mortalité et la santé humaine),

- les rayonnements ionisants (effets sur la santé humaine de l'exposition à la radioactivité non naturelle),

- la formation d'ozone photochimique (formation d'ozone et autres composés oxydants à partir de polluants primaires comme les oxydes d'azote, les composés organiques volatils non méthaniques, le monoxyde de carbone et le méthane), 
- l'acidification des terres et des eaux douces (liée aux composés soufrés, aux composés azotés et au dioxyde de carbone),

- l'eutrophisation marine (déséquilibre entre l'apport et la consommation naturelle de nutriments par l'écosystème).

Cette sélection d'indicateurs est issue d'une revue bibliographique comprenant notamment les recommandations de la Commission Européenne exprimées dans le ILCD Handbook [11], le Référentiel Alimentaire BPX 30323-1 [12] et le référentiel sous-sectoriel des boissons spiritueuses [13] issus des travaux de la plateforme française pour l'affichage environnemental des produits et la démarche européenne PEFCR en cours sur le vin [10].

La méthode ILCD 2011 a été appliquée pour le calcul de la caractérisation des impacts, sauf pour la consommation d'eau exprimée par un indicateur de consommation, ne prenant pas en compte l'eau disponible

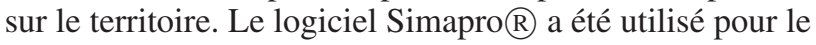
calcul des impacts.

En lien avec les recommandations de la norme ISO 14040 [1], le choix a été fait de réaliser une revue critique externe et indépendante en vue de la diffusion des résultats. $\mathrm{Au}$ moment de la rédaction du présent article, la revue critique est en cours. Les résultats présentés sont donc provisoires. Les résultats définitifs pourront être obtenus auprès des auteurs.

\section{Résultats}

\subsection{Inventaire de Cycle de Vie de la filière des Vins AOP de Beaujolais et Bourgogne}

Le premier résultat est un ICV adapté au périmètre spécifique de la filière agroalimentaire. La Fig. 2 présente les étapes du cycle de vie étudiées et les flux intermédiaires de référence pour chaque étape.

L'ICV de la filière des vins AOP de Beaujolais et Bourgogne débute par la production de 312900 tonnes de raisins de cuve (94\% en système de production intégrée et $6 \%$ en agriculture biologique), transformés ensuite en $2371000 \mathrm{hl}$ de vins. Les raisins sont vinifiés par les caves particulières (66\% du volume), les caves coopératives (30\%) et les négociants (4\%). Les sous-produits (marcs de raisins et lies de vins) sont valorisés par les distilleries. L'étape suivante, le conditionnement des vins, est réalisée par les négociants pour $52 \%$ des volumes de la filière. Une perte de volume de $0.3 \%$ de vins est considérée pendant l'étape de conditionnement [10]. Les $2363800 \mathrm{hl}$ de vins conditionnés sont distribués à $62 \%$ en France et à $38 \%$ à l'export. Les vins sont commercialisés à $43 \%$ par la grande distribution, à $35 \%$ en vente directe et à $22 \%$ par les cafés/hotels/restaurants. Un taux de perte théorique de $1 \%$ est appliqué à l'étape de distribution et de $5 \%$ à l'étape de consommation [10]. La consommation finale est estimée à $2223200 \mathrm{hl}$ de vins.

\subsection{Analyse de Cycle de Vie de la filière des Vins AOP de Beaujolais et Bourgogne}

Les résultats d'ACV sont présentés dans la Fig. 3. Les étapes les plus contributrices, sur la majorité des indicateurs, sont la phase viticole, le conditionnement et la distribution des vins.

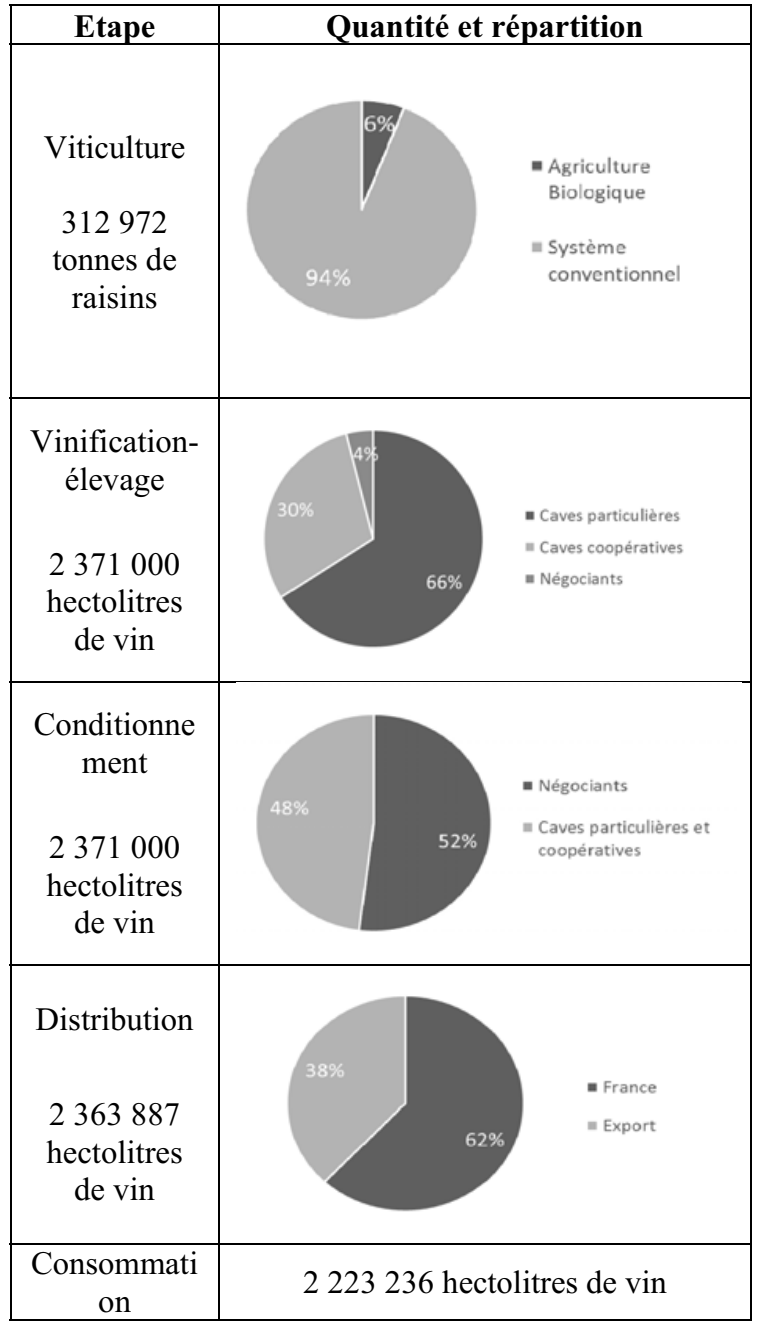

Figure 2. Schéma de synthèse des flux de produits de la filière des vins AOP de Beaujolais et Bourgogne au cours de la campagne 2011-2012.

L'impact principal de la viticulture concerne l'eutrophisation marine (61 \%), indicateur souvent utilisé pour l'étude des pratiques agricoles. L'utilisation d'amendements, dont l'azote, explique cet impact. Cette étape impacte également les indicateurs de diminution des ressources minérales et fossiles et de formation d'ozone photochimique $(31 \%)$, du fait notamment des travaux mécanisés au champ. Ces derniers, ainsi que l'application de soufre, expliquent également les impacts de la viticulture sur l'acidification (22\%). Les impacts de la phase viticole sur les autres indicateurs sont relativement plus faibles: $16 \%$ sur le changement climatique, $14 \%$ sur les émissions de particules, $6 \%$ sur la consommation d'eau et $5 \%$ sur les rayonnements ionisants.

L'étape de conditionnement des vins contribue à hauteur de $62 \%$ aux émissions de particules, $43 \%$ à l'acidification et $37 \%$ au changement climatique. La contribution aux impacts sur les autres indicateurs est légèrement plus faible : $33 \%$ sur la consommation d'eau, $30 \%$ sur la diminution des ressources minérales et fossiles, $28 \%$ sur la formation d'ozone photochimique et $11 \%$ sur les rayonnements ionisants. La fabrication des bouteilles en verre explique la majeure partie des impacts.

La contribution de la phase de distribution des vins varie peu en fonction des indicateurs: de $19 \%$ pour 


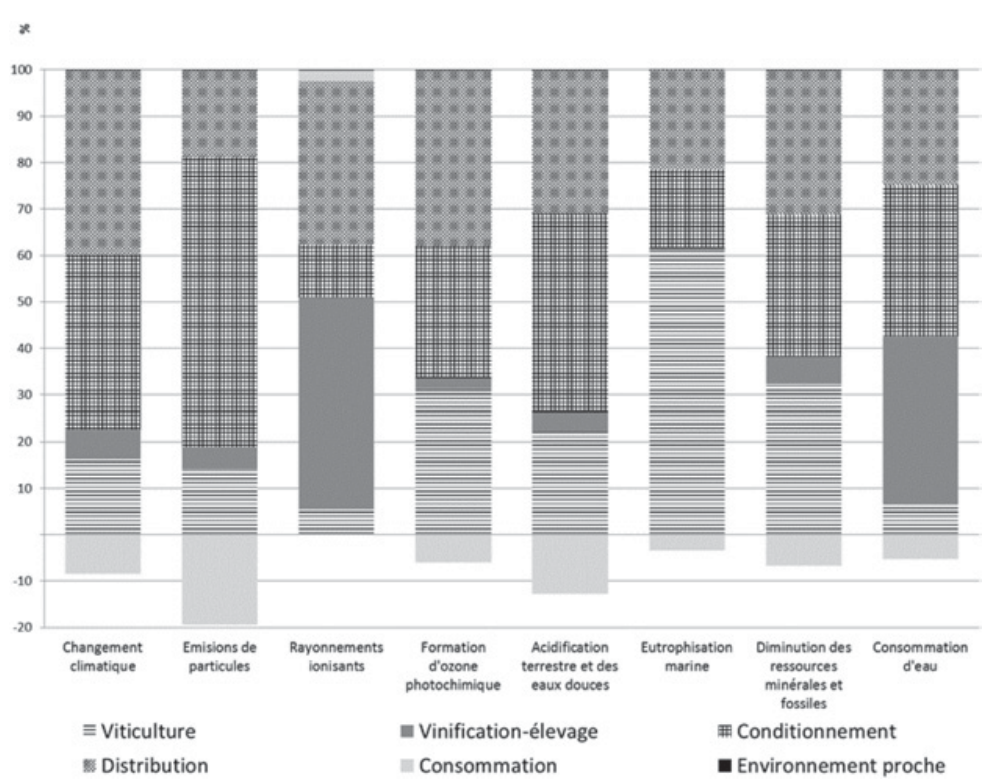

Figure 3. Impacts environnementaux potentiels de la filière des vins AOP de Beaujolais et Bourgogne au cours de la campagne 2011-2012.

les émissions de particules à $40 \%$ pour le changement climatique. Le transport des vins, selon différents modes (routier, ferroviaire et aérien) explique les impacts.

La phase de vinification-élevage contribue principalement aux impacts sur deux indicateurs : les rayonnements ionisants à hauteur de $45 \%$ et la consommation d'eau à hauteur de $36 \%$. La consommation d'électricité et d'eau au chai explique ces impacts.

La phase de consommation présente essentiellement des impacts évités, mis à part sur les rayonnements ionisants. La prise en compte d'un taux important de recyclage du verre explique les impacts évités de cette étape.

Enfin, l'environnement proche n'est que très faiblement contributeur puisque ses impacts sont inférieurs à $1 \%$ pour tous les indicateurs.

\section{Discussion}

Peu d'études prennent en compte un périmètre et une unité fonctionnelle identiques. Il est donc difficile de confronter ces résultats à d'autres.

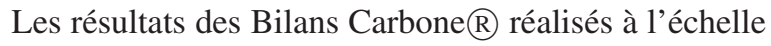
d'autres vignobles français peuvent être étudiés. Les résultats du Bilan Carbone $\mathbb{R}$ des vins de Bordeaux réalisés en 2008 sont cohérents avec ceux d'ACYDU. Ils montrent en effet que les emballages primaires (dont le verre) sont le principal contributeur à l'impact de la filière sur le changement climatique. Le deuxième poste contributeur est la distribution des vins. Enfin, le transport des personnes (dont l'oenotourisme) est le troisième poste contributeur au changement climatique. Les résultats du

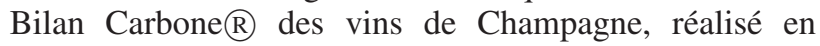
2003 et actualisé en 2015 [14], sont également cohérents avec ACYDU. Le principal contributeur $(50 \%)$ est le poste « achats et amortissements» au sein duquel $33 \%$ des impacts sont liée aux emballages. Les transports expliquent $25 \%$ à $30 \%$ des impacts. La culture de la vigne et l'élaboration du vin, considérés comme une même étape, contribuent à hauteur de $15 \%$ à $20 \%$ aux impacts de la filière sur le changement climatique.

Les ACV réalisées par Neto et al. [4] et Petti et al. [5] peuvent également être comparées. Les deux études considèrent comme périmètre la « chaîne de production » : de la viticulture à la distribution.

L'ACV réalisée en 2013 par Neto, Dias et Machado étudie la chaîne de production d'un vin blanc produit sous AOP au Portugal, le vinho verde. L'unité fonctionnelle est une bouteille de 0,751 de vihno verde. L'inventaire a été réalisé auprès d'une entreprise produisant $25 \%$ des volumes de vinho verde. La méthode utilisée pour la caractérisation des impacts est la méthode CML 2001.

Malgré ces différences, les contributions relatives des différentes étapes peuvent être comparées sur deux indicateurs : le changement climatique et l'eutrophisation marine. Les différences sont importantes puisque la viticulture est responsable d'une grande partie des impacts sur ces deux indicateurs : $68 \%$ sur le changement climatique (16\% dans le cadre d'ACYDU) et $90 \%$ sur l'eutrophisation (61 \% dans le cadre d'ACYDU). Les résultats observés par Neto et al. sont proches de ceux observés généralement dans les ACV appliquées au vin, et plus globalement aux produits agricoles.

Le décalage observé dans les résultats d'ACYDU semble pouvoir être expliqué par plusieurs facteurs. Le premier facteur est le transport des vins en avion pour la filière Beaujolais et Bourgogne $(59000 \mathrm{hl}$ en 2011-2012 soit 2,5\% du volume total distribué). Une analyse de sensibilité réalisée sur cet élément de l'ICV montre que le transport en avion augmente de $27 \%$ la contribution de l'étape de distribution sur l'indicateur de changement climatique et de $33 \%$ l'impact global de la filière sur cet indicateur.

D'autre part, le transport des raisins entre la parcelle et le chai de vinification est intégré à l'étape de viticulture dans l'étude de Neto et al., alors qu'ACYDU considère qu'elle est intégrée dans l'étape de vinification-élevage. Le transport de la récolte explique en effet $90 \%$ des impacts 
du poste Transports de cette étape sur le changement climatique.

De plus, dans le projet ACYDU, une hypothèse a été formulée pour prendre en compte les déplacements des salariés en charge de la commercialisation des vins. Au nombre de 1280, ils parcourent plus de 76 millions de $\mathrm{km}$. Cet impact est associé à l'étape de distribution. Une analyse de sensibilité montre que la non prise en compte de ces déplacements professionnels engendre une diminution de $6 \%$ à $81 \%$ selon les indicateurs des impacts de l'étape de distribution (dont $16 \%$ pour l'indicateur de changement climatique).

Enfin, le dernier facteur explicatif est la représentativité des données. En effet, bien que basé sur les données d'une seule entreprise, l'inventaire de l'étude de Neto et al. est représentatif de $25 \%$ du volume produit dans l'appellation. Malgré un nombre important d'entreprises enquêtées (17), la représentativité de l'échantillon d'ACYDU sur les volumes produits n'atteint pas ce score. De plus, l'enquête réalisée dans le cadre d'ACYDU n'a pas porté sur la phase viticole pour laquelle la base de données AGRIBALYSE $\mathbb{R}$ a été mobilisée.

En 2006, Petti et al. publient une ACV de la « chaîne de production » (de la viticulture à la distribution du vin) d'un vin produit en agriculture biologique, sous appellation, en Italie. L'inventaire est réalisé auprès d'une exploitation de 12 ha. L'unité fonctionnelle et la méthode de caractérisation sont les mêmes que dans l'étude de Neto et al. : 0,751 de vin et méthode CML 2001. Les résultats obtenus sont plus proches de ceux d'ACYDU puisque la fabrication des intrants de conditionnement apparait comme le principal contributeur aux impacts sur plusieurs indicateurs, dont le changement climatique à hauteur de $62 \%$. Le deuxième contributeur est la distribution des vins (16\%), suivie par la fabrication des emballages secondaires $(10 \%)$. La phase viticole n'apparait qu'en quatrième position $(9 \%)$.

Ces deux études [4,5] intègrent dans une même étape la fabrication et la fin de vie des bouteilles en verre, alors qu'ACYDU attribue leur fabrication à l'étape de conditionnement et leur fin de vie à l'étape de consommation. Les impacts de l'étape de consommation sont des impacts évités pour 7 indicateurs sur 8 . La fin de vie des bouteilles de verre (intégrant un taux de $85 \%$ de recyclage) explique ces impacts évités. L'intégration de la fin de vie des bouteilles à la phase de conditionnement aurait alors pour conséquence la diminution de la contribution de l'étape de conditionnement sur 7 indicateurs (dont environ moins $20 \%$ sur les émissions de particules, moins $12 \%$ sur l'acidification et moins $8 \%$ sur le changement climatique).

L'établissement d'un Inventaire de Cycle de Vie à l'échelle d'une filière agricole est une tâche complexe. La quantification des flux au niveau des étapes puis à une échelle plus globale nécessite un travail important de recherche et de compilation de données. Malgré les efforts mis en œuvre pour assurer une bonne représentativité des données, la collecte de données pour ce type de projets dépend des réponses obtenues par les différentes entreprises qui le font sur le mode du volontariat. D'autre part, la multiplicité des structures de petites tailles dans le secteur viti-vinicole français rend la tâche encore plus difficile. Ainsi, même si 17 entreprises ont participé au projet, elles ne représentent que $9 \%$ du vin produit sur
Tableau 3. Comparaison de la répartition géographique des volumes produits à l'échelle de la filière et au sein de l'échantillon.

\begin{tabular}{|l|c|c|}
\hline & $\begin{array}{c}\text { Beaujolais } \\
(\mathbf{\%})\end{array}$ & $\begin{array}{c}\text { Bourgogne } \\
(\mathbf{\%})\end{array}$ \\
\hline $\begin{array}{l}\text { Filière des vins } \\
\text { AOP de Beaujolais et } \\
\text { Bourgogne en 2011-2012 }\end{array}$ & 36 & 64 \\
\hline $\begin{array}{l}\text { Echantillon de 17 } \\
\text { entreprises }\end{array}$ & 40 & 60 \\
\hline
\end{tabular}

le territoire. La répartition géographique (entre Beaujolais et Bourgogne) des entreprises de l'échantillon semble cependant représentative de la filière (cf. Tableau 3).

\section{Conclusion}

Les travaux menés dans le cadre du projet ont permis (i) de proposer une méthodologie pour la construction d'un ICV qui soit adapté au périmètre d'une filière agroalimentaire et dont le niveau de qualité des données est élevé ; et (ii) de mettre en évidence les impacts environnementaux de la filière Vins AOP de Beaujolais et Bourgogne, apportant ainsi un éclairage pour la définition des axes stratégiques pour l'avenir.

\section{Références}

[1] ISO. 2006a. ISO 14040 - environmental management - life cycle assessment - principles and framework

[2] ISO. 2014. ISO/TS 14072 - environmental management - life cycle assessment - requirements and guidelines for Organizational Life Cycle Assessment

[3] E. Loiseau, G. Junqua, P. Roux, V. Bellon-Maurel, Environmental assessment of a territory : an overview of existing tools and methods, Journal of Environmental Management, Vol 112 (2012)

[4] B. Neto, A.C. Dias, M. Machado. 2013, Life cycle assessment of the supply chain of a Portuguese wine: from viticultire to distribution, The International Journal of Life Cycle Assessment, Vol. 18 (2013)

[5] L. Petti, A. Raggi, C. De Camillis, P. Matteucci, B. Sara, G. Pagliuca, Life cycle approach in an organic wine-making firm: an Italian case study, Fifth Australian Conference on Life Cycle Assessment, Melbourne, Australia (2006)

[6] V. Lempereur, S. Penavayre, G. Assogba, T. Majchrzak, C. Pernet, Méthodologie de description d'une filière. Application à la filière viti-vinicole du Beaujolais et de Bourgogne. $38^{\text {ème }}$ Congrès Mondial de la Vigne et du Vin et $13^{e ̀ m e}$ Assemblée Générale de l'Organisation Internationale de la Vigne et du Vin (2015)

[7] G. Assogba, Evaluation des dimensions, sociales, environnementales et territoriales de filières agroalimentaires sous signes officiels de qualités. Discussion des méthodes et des premiers résultats du projet ACYDU appliqué aux filières IGP Canards Gras du Sud-Ouest, AOP Comté et AOP Vins de Bourgogne. $52^{\text {ème }}$ colloque ASRDLF (2015)

[8] A. Besnier, L. Farrant, S. Penavayre, F. Bosque, M.P. Labau, V. Lempereur, C. Pernet, J.L. Berner, 
F. Jolibert, LCI of products sectors : from field data collection to the big picture, $10^{\text {th }}$ International conference on Life Cycle Assessment of Food, Dublin (2016)

[9] P. Koch, T. Salou, V. Colomb, S. Payen, S. Perret, A. Tailleur, S. Willmann, 2015. Agribalyse: rapport méthodologique. Version 1.2 (2014)

[10] Comité Européen des Entreprises du Vin, PEFCR pilot on wine - PEF screening report in the context of the EU Product Environmental Footprint Category Rules (PEFCR) Pilots (2016)
[11] JRC, European Commission, ILCD Handbook (2011)

[12] ADEME/AFNOR, Référentiel transversal d'évaluation de l'impact environnemental des produits alimentaires (2012)

[13] Fédération Française des Spiritueux, Projet de référentiel catégoriel concernant l'évaluation environnementale des boissons spiritueuses en vue de l'affichage environnemental des produits (2016)

[14] Comité Champagne, Climat : la Champagne responsable et engagée, Dossier de presse (2015) 